\title{
The Fourth Circle
}




\section{EAST-WEST CENTER \\ SERIES ON

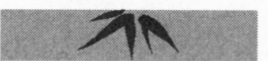 \\ CONTEMPORARY ISSUES IN ASIA AND THE PACIFIC}

Series Editor, Muthiah Alagappa 


\section{The Fourth Circle}

A POLITICAL ECOLOGY OF SUMATRA'S

RAINFOREST FRONTIER

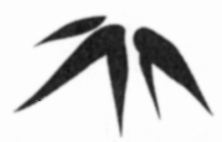

John F. McCarthy

Stanford University Press

Stanford, California

2006 
(C) 2006 by the Board of Trustees of the

Leland Stanford Junior University.

All rights reserved.

No part of this book may be reproduced or transmitted in any form or by any means, electronic or mechanical, including photocopying and recording, or in any information storage or retrieval system without the prior written permission of Stanford University Press.

Library of Congress Cataloging-in-Publication Data

McCarthy, John F. (John Fitzgerald), I964-

The fourth circle : a political ecology of Sumatra's rainforest frontier / John F. McCarthy.

p. $\quad \mathrm{cm} .-$ (Contemporary issues in Asia and the Pacific)

Includes bibliographical references and index.

ISBN 0-8047-52 I I-7 (cloth : alk. paper)

ISBN 0-8047-52 I 2-5 (pbk. : alk. paper)

I. Forest policy-Indonesia-Nanggroe Aceh Darussalam.

2. Forest management-Indonesia-Nanggroe Aceh Darussalam. 3. Biological diversity conservation-Indonesia-Nanggroe Aceh Darussalam. 4. Farms, SmallIndonesia-Nanggroe Aceh Darussalam. 5. Adat law-Indonesia-Nanggroe Aceh

Darussalam. I. Title. II. Series.

$$
\begin{gathered}
\text { SD657.I5M37 } 2006 \\
333.7509598^{\prime} \mathrm{I}-\mathrm{dc22} \\
2005025804 \\
\text { Original Printing } 2006
\end{gathered}
$$

Last figure below indicates year of this printing:

$$
\begin{array}{llllllllll}
\text { I } 5 & \text { I4 } & \text { I3 } & \text { I2 } & \text { II } & \text { IO } & 09 & 08 & 07 & 06
\end{array}
$$

